\title{
Primary and secondary cosmic rays in the NUCLEON space experiment after two years of data acquisition
}

\section{A. Panov*}

Skobeltsyn Institute of Nuclear Physics, Moscow State University, Moscow, Russia

E-mail: panovedec1.sinp.msu.ru

\section{E. Atkin}

National Research Nuclear University "MEPhI", Moscow, Russia

E-mail: evatkin@mephi.ru

\section{N. Gorbunov}

Joint Institute for Nuclear Research; Dubna State University, Dubna, Russia

E-mail: gorbunovesunse.jinr.ru

\section{Grebenyuk}

Joint Institute for Nuclear Research; Dubna State University, Dubna, Russia

E-mail: greben@jinr.ru

\section{Karmanov}

Skobeltsyn Institute of Nuclear Physics, Moscow State University, Moscow, Russia E-mail: karmanov68@mail.ru

\section{Kovalev}

Skobeltsyn Institute of Nuclear Physics, Moscow State University, Moscow, Russia E-mail: im.kovalev@physics.msu.ru

\section{Kudryashov}

Skobeltsyn Institute of Nuclear Physics, Moscow State University, Moscow, Russia E-mail: ilya.kudryashov.85@gmail.com

\section{A. Kurganov}

Skobeltsyn Institute of Nuclear Physics, Moscow State University, Moscow, Russia E-mail: meess107.ru

\section{Merkin}

Skobeltsyn Institute of Nuclear Physics, Moscow State University, Moscow, Russia E-mail: merkinmesilab.sinp.msu.ru

\section{Podorozhny}

Skobeltsyn Institute of Nuclear Physics, Moscow State University, Moscow, Russia E-mail: dmp@eas.sinp.msu.ru

\section{S. Porokhovoy}

Joint Institute for Nuclear Research, Dubna, 141980, Russia

E-mail: porokhenusun.jinr.ru 


\section{Shumikhin}

National Research Nuclear University “MEPhI”, Moscow, Russia

E-mail: shuma.v.v@mail.ru

\section{A. Tkachenko}

Joint Institute for Nuclear Research, Dubna, Russia

E-mail: avtajinr.ru

\section{Tkachev}

Joint Institute for Nuclear Research; Dubna State University, Dubna, Russia

E-mail: tkatchevejinr.ru

\section{A. Turundaevskiy}

Skobeltsyn Institute of Nuclear Physics, Moscow State University, Moscow, Russia

E-mail: ant@eas.sinp.msu.ru

\section{O. Vasiliev}

Skobeltsyn Institute of Nuclear Physics, Moscow State University, Moscow, Russia

E-mail: oav@rsx.sinp.msu.ru

\section{A. Voronin}

Skobeltsyn Institute of Nuclear Physics, Moscow State University, Moscow, Russia

E-mail: voroninesilab.sinp.msu.ru

The NUCLEON space experiment was designed to measure energy spectra of cosmic ray nuclei from protons to iron in the energy range approximately from $1 \mathrm{TeV}$ per particle to $1 \mathrm{PeV}$ per particle with good individual charge resolution. The apparatus started the collection of data at the beginning of 2015 and it is in operation up to now. The duration of the mission is expected to be no less than five years. One of the main feature of the experiment is an implementation of two different methods of energy measurement on board of the apparatus - the calorimetric method and the new kinematic KLEM method (Kinematic Lightweight Energy Meter). The data obtained after two years of operation are presented. A multiple signs of complex behavior of the nuclei spectra and the ratios of spectra violating of a simple universal power-law behavior of the spectra will be presented in the report.

35th International Cosmic Ray Conference - ICRC2017

10-20 July, 2017

Bexco, Busan, Korea

\footnotetext{
*Speaker.
} 


\section{Introduction}

The space experiment NUCLEON was implemented within a long-standing tradition of investigation the physics of cosmic rays in Skobeltsyn Institute of Nuclear Physics of Lomonosov Moscow State University (SINP MSU). The prehistory of the NUCLEON experiment may be started with the invention of ionization calorimeter by N.L. Grigorov, V.S. Murzin and I.D. Rapoport in $1958[1,2]$. The first direct measurements of the cosmic-ray energy spectrum in open space were experiments on the Proton series satellites (Proton-1, Proton-2, Proton-3, Proton-4) in 1965-1968 [3, 4], which were developed in SINP MSU on the base of using an ionization calorimeter. These experiments were not only the first direct experiments with using of ionization calorimeter in the space, but, in a certain sense, they were paradigmatic examples for the extremely wide class of balloon and space spectrometers that followed them, including those currently working in the space and planned for the foreseeable future. Proton-4 mission measured all-particle spectrum up to the knee energy region [4] which is the best result in the direct measurements of cosmic rays up to now. In 1984-1986 two space spectrometers developed in SINP MSU Sokol-1 and Sokol-2 based on deep ionization calorimeters measured spectra of cosmic ray nuclei group and the all-particle spectrum to energies about $100 \mathrm{TeV}$ per particle [5, 6, 7, 8, 9]. Finally, SINP MSU participated in the international balloon experiment ATIC that had three successful Antarctic lights in 2000-2008 and provided many results both in measurement of spectra of cosmic ray nuclei $[10,11,12]$ and cosmic ray electrons $[13,14]$. The NUCLEON experiment was developed on the basis of the experience gained in all these earlier works, and within the scientific school that was developed during their implementation.

One of the most notable features in the energy spectrum of cosmic rays is the sharp increase in the slope of the energy spectrum near $3 \times 10^{15} \mathrm{eV}(3 \mathrm{PeV})$ per particle - the so-called "knee." The nature of this "knee" is still unclear, and represents one of the major mysteries of cosmic ray physics and space physics in general. The "knee" in the spectrum of cosmic rays has been found and is still observed in the EAS (extensive air showers) experiments, which provide data on the energy spectrum of cosmic rays at very high energies, but do not give reliable information about their chemical composition. At the same time, for understanding the physics near the "knee," it would be very important to know the behavior of the individual components of the flux of cosmic rays near this area. Much more detailed information on the chemical composition of cosmic rays is provided by direct space of balloon experiments, in which the spectrometer is moved out of the atmosphere, where cosmic-ray particles can be observed directly, using different types of spectrometers. One of the main tasks for modern cosmic-ray direct experiments is the study of chemical composition near the knee region.

Even in the energy region below $1 \mathrm{PeV}$ per particle there are signatures of complex behavior of cosmic-ray spectra. As an example of such signature we can point out the spectrum of cosmic-ray protons in the energy region about $10-20 \mathrm{TeV}$. A compilation of the data before the NUCLEON experiment and before very recent data of the CREAM-III experiment [15] is shown in figure 1. A sign of a break is seen in the spectrum near the energy $10 \mathrm{TeV}$. However none of the previous experiments showed statistically significant confirmation of existence of this feature. Obviously, this region of the spectrum should be urgently inspected with higher statistics and reliability. Another example is observations of hardening (upturn) of the spectra of heavy nuclei near the energy 


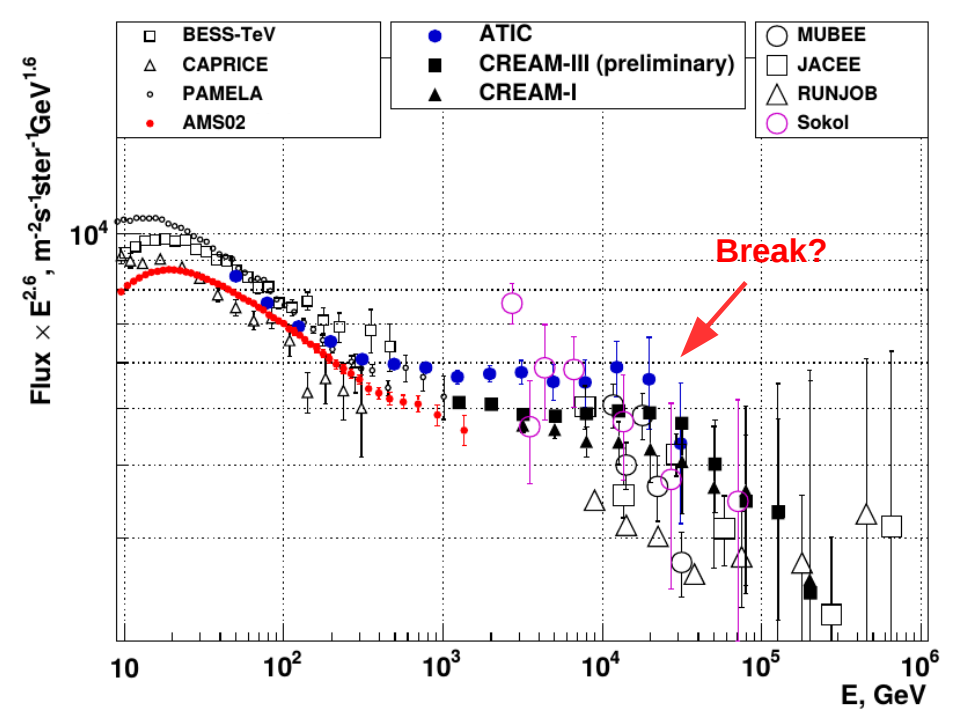

Figure 1: A compilation of the data on proton spectrum before the NUCLEON experiment and a very recend data of CREAM experiment [15]. The shown spectra are: AMS02 [17], BESS-TeV [18, 19, 20], CAPRICE [21], PAMELA [22], ATIC [11], CREAM-III (preliminary) [23], CREAM-I [24], MUBEE [25, 26], JACEE [27], RUNJOB [28].

$\sim 10 \mathrm{TeV}$ per particle, that corresponds to the energy of a few hundred $\mathrm{GeV}$ per nucleon, observed in ATIC and CREAM experiments $[10,16]$. The details of behavior of the heavy nuclei spectra at energies higher than $100 \mathrm{TeV}$ per particle are almost completely unknown and might contain interesting features. In this paper we present the latest data of the direct NUCLEON space experiment on spectra of both primary and secondary nuclei from protons to iron up to the energies of hundreds TeVs per particle.

\section{The instrument and data taking}

The NUCLEON space experiment was designed mainly to measure the spectra of cosmic ray nuclei with an individual charge resolution in the energy range from a few $\mathrm{TeV}$ to $1 \mathrm{PeV}$ per particle, while having a lower energy threshold of a few hundred GeV. The most important feature of the NUCLEON detector is the implementation of two different particle energy measurement methods: the first uses an ionization calorimeter, and the second is a kinematic method, the Kinematic Lightweight Energy Meter (KLEM) [29, 30, 31, 32, 33], which is based on the measurement of the multiplicity of secondary particles after the first nuclear interaction of a primary particle with a target of the spectrometer. The advantage of the KLEM method compared to the conventional calorimetric method is the ability to provide a high aperture of the device with a low weight of the equipment. The presence of two methods of energy measurement in the NUCLEON detector will allow studying and calibrating the new KLEM method using a conventional calorimetric method.

The main systems of the spectrometer (see figure 2) are four planes of the charge measurement system (ChMS), a carbon target, six planes of the energy measurement system using the KLEM method (KLEM system tracker), three double-layer planes of the scintillator trigger sys- 


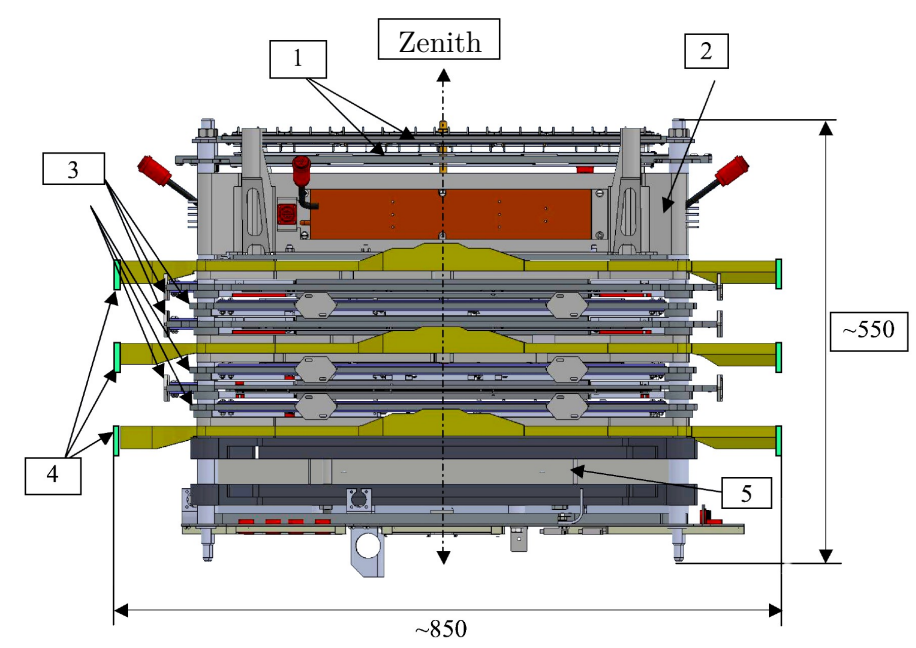

Figure 2: The NUCLEON detector simplified scheme. 1 - charge measurement system (ChMS), 2 - carbon target, 3 - KLEM system tracker, 4 - scintillator trigger system, 5 - ionization calorimeter.

tem, and a small aperture calorimeter (IC). Details of the detector design are provided in the articles $[34,35,36,37,38]$. The weight of the detector is approximately $360 \mathrm{~kg}$; the power consumption does not exceed $160 \mathrm{~W}$. On December 28, 2014, the NUCLEON detector was launched into a sun-synchronous orbit with an average altitude of $475 \mathrm{~km}$ and an inclination of 97 degrees as an additional payload of the Russian satellite Resource-P 2. On January 11, the NUCLEON detector was powered and started to collect data. The planned lifetime of the NUCLEON detector is at least five years. Some details of the implementation of the methods of energy measurement with KLEM and calorimetric methods in the NUCLEON experiment are given in the paper [39].

In this report we present and discuss the data related for the period of data taking of the NUCLEON experiment from July 2015 to the end of June 2017. There were 334 days of pure astronomical time of the data taking that correspond to 218 days of the apparatus live time. There was eight months delay in data acquisition in 2015-2016 due to the solution of the main task of the Resource-P 2 serial satellite was incompatible with the operation of the NUCLEON observatory as an additional payload.

\section{Results and discussion}

\subsection{Protons and helium spectra}

Figure 3 shows the energy spectra of protons and helium nuclei obtained by the NUCLEON experiment both with the KLEM and with the calorimeter methods. There are no strong deviations from the results of the other experiments (see the caption under the pictures). The results of the KLEM method and the calorimetric method are in good agreement. The statistics of the KLEM method are approximately two time greater the statistics of the calorimetric method due to larger geometric factor of the KLEM tracker compared to the ionization calorimeter.

The ratio of the protons to helium spectra are shown in figure 4 . The left panel of the figure 4 demonstrates the expected decreasing of $\mathrm{p} / \mathrm{He}$ ratio with energy increasing. This effect was estab- 

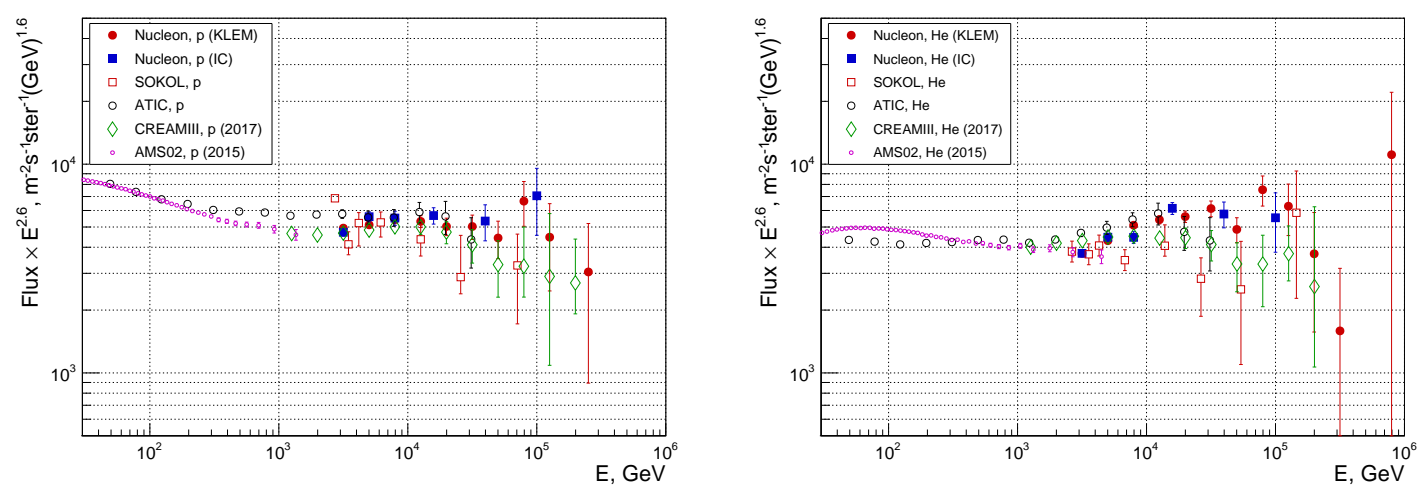

Figure 3: Energy spectra of protons and helium nuclei obtained by the NUCLEON experiment by the KLEM method (red circles) and by the calorimeter method (blue squares) and in the experiments SOKOL [9], ATIC [11], CREAMIII [15], AMS-02 [17].
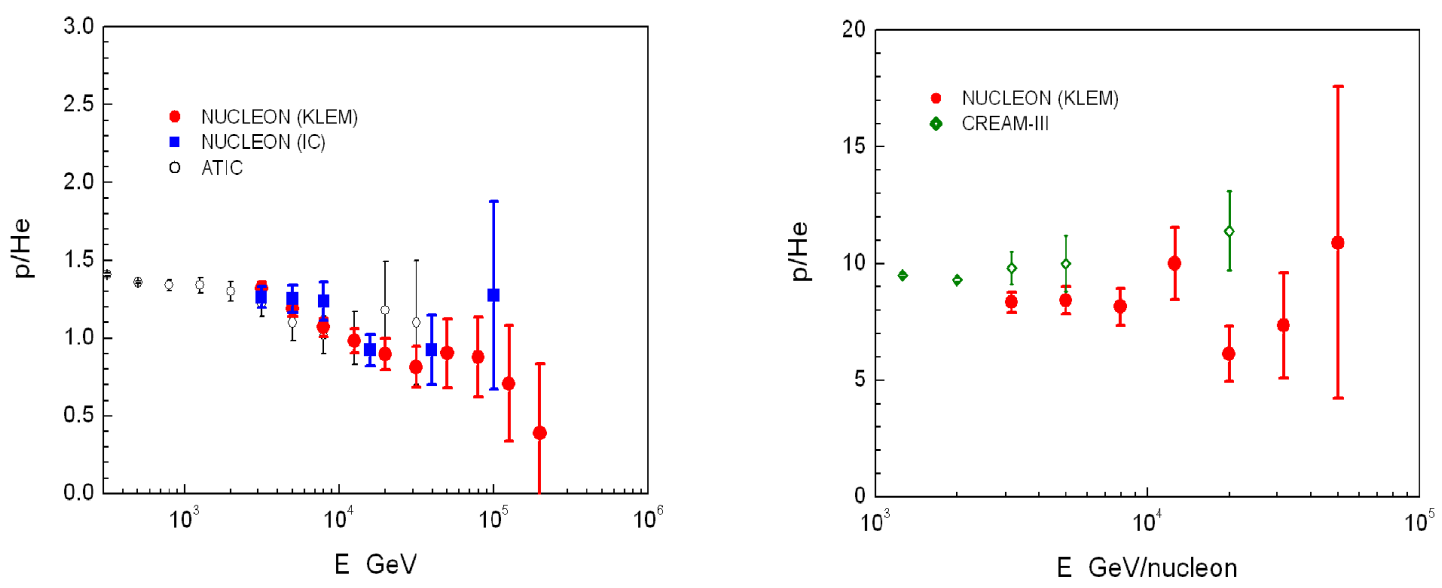

Figure 4: The ratio of the protons to helium spectra. Left panel - in terms of energy per particle; right panel - in terms of enery per nucleon. The data of NUCLEON are compared to the data of ATIC [11] and CREAM-III [15].

lished for the first time with high statistical confidence in the ATIC experiment [40] in 2004 up to energies about $10 \mathrm{TeV}$ and was confirmed at these energies in a number of other experiments. The NUCLEON experiment studies this ratio up to the energies higher than $100 \mathrm{TeV}$. The decreasing of the ratio is generally confirmed, with the exception of the highest energies, in which there are signs of violation of this law. The NUCLEON data is compared with the data of the ATIC experiment [11] in the right panel of the figure 4 in terms of the energy per particle. There is a good agreement between NUCLEON and ATIC data. The data of NUCLEON in the terms of energy per nucleon are compared with the recent data of the CREAM-III experiment [15]. The data are related to the highest end of the studied region. There is a good agreement between NUCLEON and CREAM-II results, and both experiments point out that the spectra of protons and helium have approximately same slopes at such energies.

The spectra of protons and helium in terms of magnetic rigidities of the present work and 

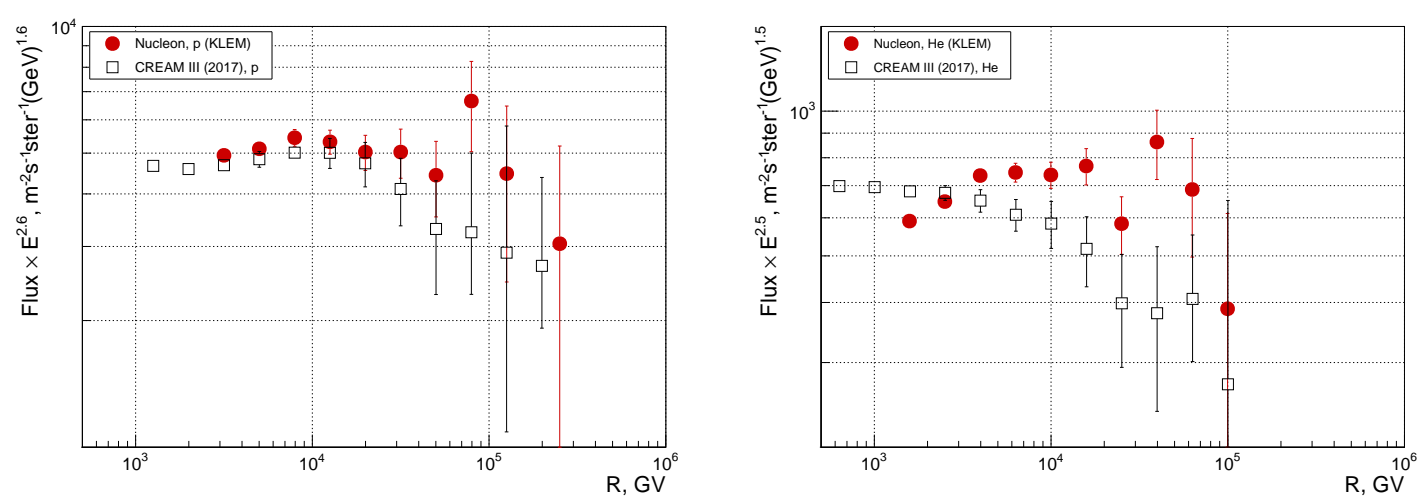

Figure 5: The spectra of protons and helium in terms of magnetic rigidities. Present work (KLEM method) and CREAM-III [15] data are shown.

CREAM-III [15] are shown in figure 5. Only the spectra measured by the KLEM method are shown for the NUCLEON experiment since the spectra of calorimeter are the same within the statistical errors, but the statistics of the KLEM spectra is better. There is a sign of a break of the spectra near the rigidity $\sim 10 \mathrm{TV}$. This break is seen both in the spectra of protons and helium and confirms by the data of NUCLEON and CREAM-III. This feature of the protons and helium spectra was discussed in recent papers of NUCLEON [39] and CREAM-III [15]. The statistical significance of the effect is not discussed here. Let us emphasis that the are some systematic difference between the spectra measured by NUCLEON and by CREAM-III (especially for helium nuclei) but both experiments point out to the existence of a break. In the case of the helium spectrum the energy of the break looks somewhat lower than the energy of the break in the spectrum of protons, but this may be illusion of the statistics. This question should be studied more carefully with higher statistics.

\subsection{Abundant heavy nuclei}

Figure 6 shows the energy spectra of carbon and oxygen nuclei obtained by the NUCLEON experiment both for the KLEM and the calorimeter methods. There are no strong deviations from the data of other experiments (see the caption under the pictures). Some differences between the calorimeter and the KLEM methods are seen for the carbon spectrum, but for oxygen spectrum the data of KLEM and calorimeter are in good agreement. If the differences in carbon data would be systematic, than one should expect similar differences in the oxygen spectrum, but actually it is not the case. Therefore we can not confidently relate the differences of the results in the carbon spectrum to a systematic origin; the origin of this discrepancy has been studied.

The spectra of carbon and oxygen are hard above energy $2 \mathrm{TeV}$ per particle. From the KLEM data $\gamma_{C}=2.381 \pm 0.033, \gamma_{O}=2.442 \pm 0.039$; from the calorimeter data $\gamma_{C}=2.269 \pm 0.042, \gamma_{O}=$ $2.410 \pm 0.035$. It is seen that the spectrum of carbon may be harder than the spectrum of oxygen, but the statistical significance of this conclusion is not high: $1.2 \sigma$ and $2.6 \sigma$ for the KLEM and calorimeter spectra respectively.

Figure 7 shows the ratio of the carbon spectrum to the oxygen spectrum in the terms of energy per nucleon. A sign of more hard spectrum of carbon also seen in this ratio, but the statistical 

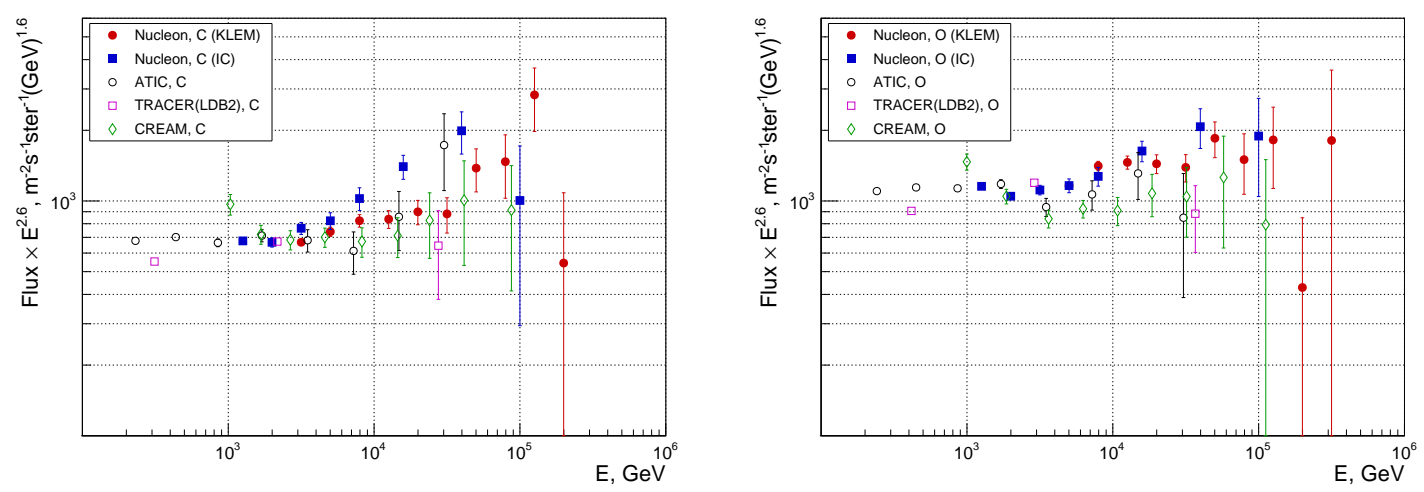

Figure 6: Energy spectra of carbon and oxygen nuclei obtained by the NUCLEON experiment and in the experiments ATIC [11], TRACER(LDB2) [41], and CREAM [16].

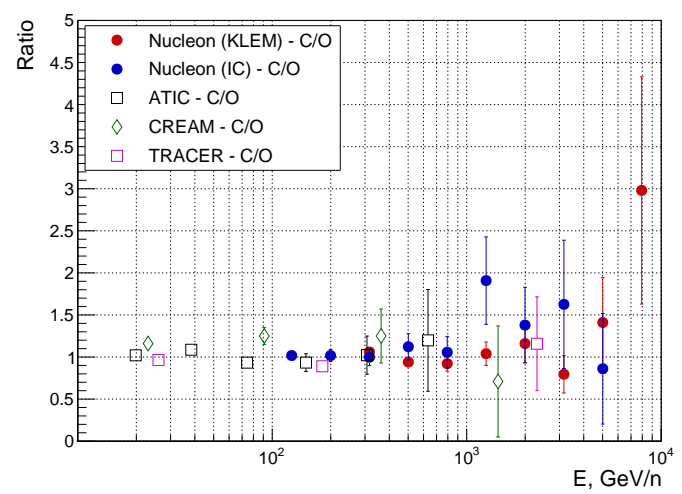

Figure 7: Energy spectra of carbon and oxygen nuclei obtained by the NUCLEON experiment and in the experiments ATIC [11], CREAM [16], and TRACER(LDB2) [41].

significance is not high again. A difference of the slopes of the carbon and oxygen spectra may be important for understanding the mechanism of cosmic ray acceleration since currently all models predict essentially same slopes for carbon and oxygen up to very small propagation effects.

Figure 8 shows the spectra of $\mathrm{Ne}, \mathrm{Mg}, \mathrm{Si}$. The spectral indices of the spectra obtained for the calorimeter method are respectively: $\gamma_{\mathrm{Ne}}=2.391 \pm 0.046, \gamma_{\mathrm{Mg}}=2.544 \pm 0.041, \gamma_{\mathrm{Si}}=2.536 \pm$ 0.038. The spectrum of $\mathrm{Ne}$ is harder than the spectra of $\mathrm{Mg}$ and $\mathrm{Si}$ with statistical significance $2.5 \sigma$ and $2.4 \sigma$ respectively. The linear approximation of the charge - spectral index relation shows (Figure 8, the bottom right plot) that the trend of the slope of spectra in the group Ne-Mg-Si exists with the statistical significance $2.3 \sigma$. The KLEM method produces lower significance of $\mathrm{Ne}-\mathrm{Mg}$ $\mathrm{Si}$ data because of higher energy threshold of the KLEM method for very heavy nuclei in the comparison with the calorimeter method, so the KLEM data do not considered here.

Figure 9 shows the spectrum of iron in the terms of energy per particle and the ratio of the spectrum of all nuclei $6 \leq Z \leq 14$ to the spectrum of iron in the terms of energy per nucleon. The spectral index of the iron spectrum from the calorimeter data is $\gamma_{\mathrm{Fe}}=2.523 \pm 0.023$. Generally, the spectrum of iron is steeper than the spectra of more light abundant heavy nuclei. The spectrum of 

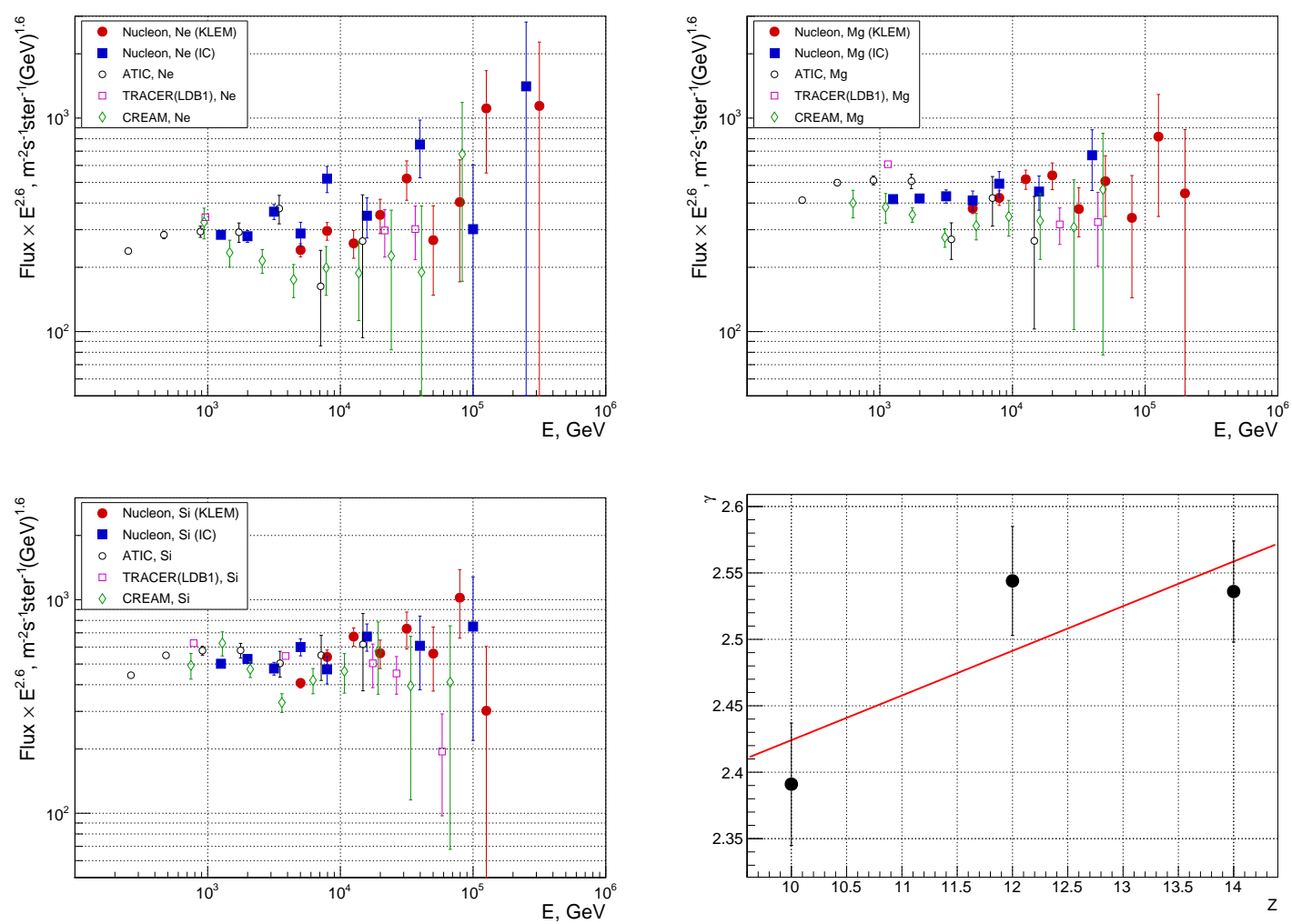

Figure 8: Energy spectra of $\mathrm{Ne}, \mathrm{Mg}$, Si obtained by the NUCLEON experiment and in the experiments ATIC [11], TRACER(LDB2) [41], and CREAM [16]. Right bottom panel: spectral index versus charge plot for $\mathrm{Ne}, \mathrm{Mg}$, Si spectra.
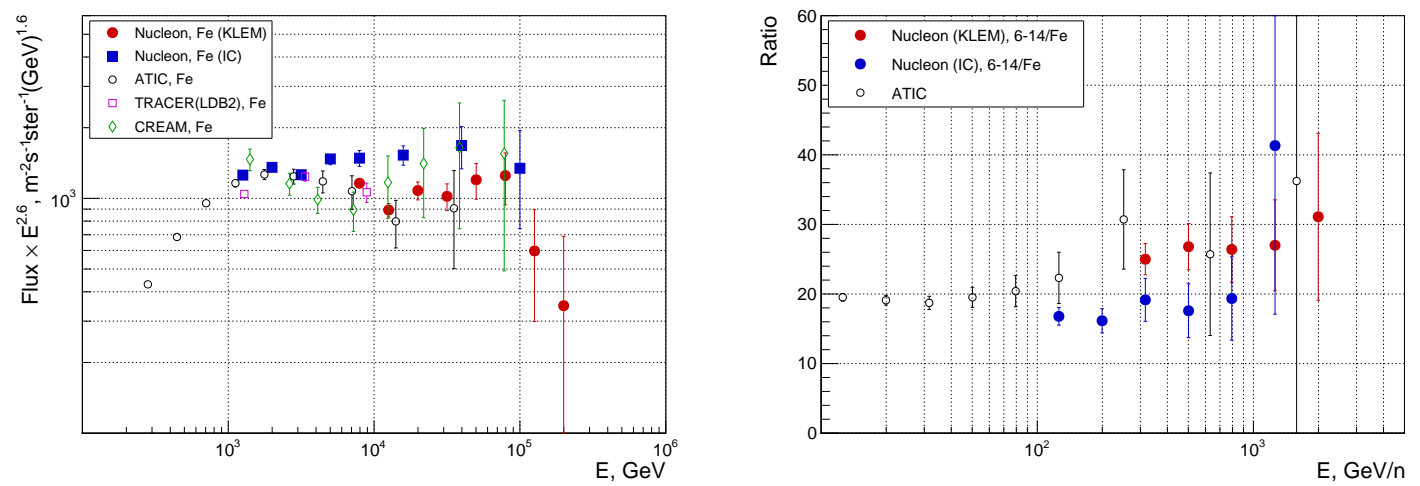

Figure 9: Left: energy spectra of iron obtained by the NUCLEON experiment and in the experiments ATIC [11], TRACER(LDB2) [41], and CREAM [16]. Right: ratio of the spectrum of all nuclei $6 \leq Z \leq 14$ to the spectrum of iron in the terms of energy per nucleon obtained by the NUCLEON experiment and by ATIC [42]. 
iron is steeper than the spectra of carbon and oxygen with the statistical significance $5.0 \sigma$ and $2.7 \sigma$ respectively. The ratio of the spectrum of all nuclei $6 \leq Z \leq 14$ to the spectrum of iron (figure 9 , right panel) shows qualitatively more steeper character of the iron spectrum than the spectra of other abundant heavy nuclei. Note, already the ATIC experiment [42] gave and indication of the same effect (see figure 9) but with low statistical reliability.
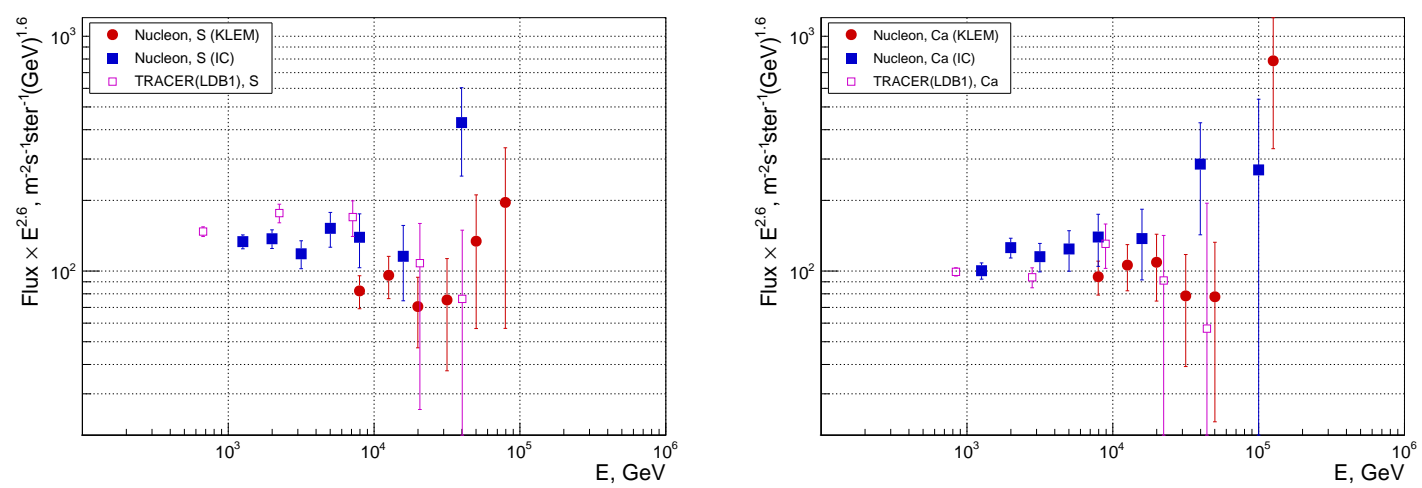

Figure 10: Energy spectra of $\mathrm{S}$ and Ca obtained by the NUCLEON experiment and in the experiment TRACER(LDB1) [43].

An interesting question is what is the character of the transition from the flat spectra of carbonsilicon group to the steeper spectrum of iron. The answer may be found from the spectra of nuclei with intermediate charges, $\mathrm{S}$ and $\mathrm{Ca}$. This spectra are shown in figure 10. However the statistics is too low yet to draw some definite conclusions.

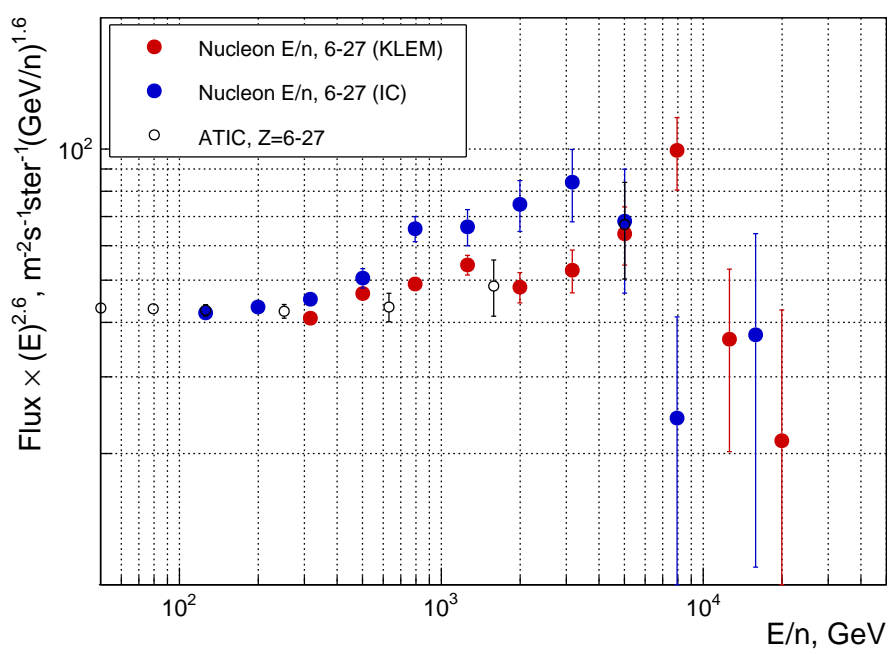

Figure 11: Spectra of heavy nuclei $(Z=6 \div 27)$ in terms of energy per nucleon from the NUCLEON experiment along with similar data from the ATIC experiment [44].

\subsection{Is there an universal break in the spectra of cosmic ray nuclei near $R=10 \mathrm{TV}$ ?}

The spectrum of all nuclei from carbon to iron in the terms of energy per nucleon measured 
in the NUCLEON experiment and in the ATIC experiment [44] is shown in figure 11. It is seen that after the hard part of the spectrum below the energy $5 \mathrm{TeV} /$ nucleon there is a break of the spectrum. The magnetic rigidity of the break is approximately $10 \mathrm{TV}$ and it is very close to the rigidity of similar breaks in the spectra of protons and helium measured (see figure 5). This may be an indication of existence of a universal break in the spectra of all cosmic ray nuclei near the magnetic rigidity of $10 \mathrm{TV}$. In fact, the universal break near $10 \mathrm{TV}$ in all cosmic ray spectra and particularly in the spectra of heavy nuclei observed by the NUCLEON experiment was predicted by the three-component model of cosmic ray spectra of Zatsepin and Sokolskaya [45]. The break is an indication of existence of a special type of cosmic ray sources with the maximal acceleration energy of order $10 \mathrm{TV}$ (the exact value in [45] is $50 \mathrm{TV}$, which produce visually a break in spectra near $10 \mathrm{TV}$ ). This type of sources was associated in [45] with supernova explosions into regular interstellar media (other two kinds of sources in the model were explosions of SN into super-bubble media and novae stars).

\subsection{All-particle spectrum}

All-particle spectrum measured by the NUCLEON experiment with the KLEM and the calorimeter methods and by the experiments PROTON-4 [4], SOKOL [8], ATIC [11] is shown in figure 12. The NUCLEON data are in good agreement with the data of other experiments except somewhat lower intensity of the data of old PROTON-4 experiment. One can note a deficit of high-energy events (above $100 \mathrm{TeV}$ ) in the NUCLEON data, both in the KLEM and calorimetric methods. The statistics in this region are not very high yet so it would be too early to say about real break in the all particle spectrum above energy $100 \mathrm{TeV}$. The situation expected to be clarified with growing up of the statistics.

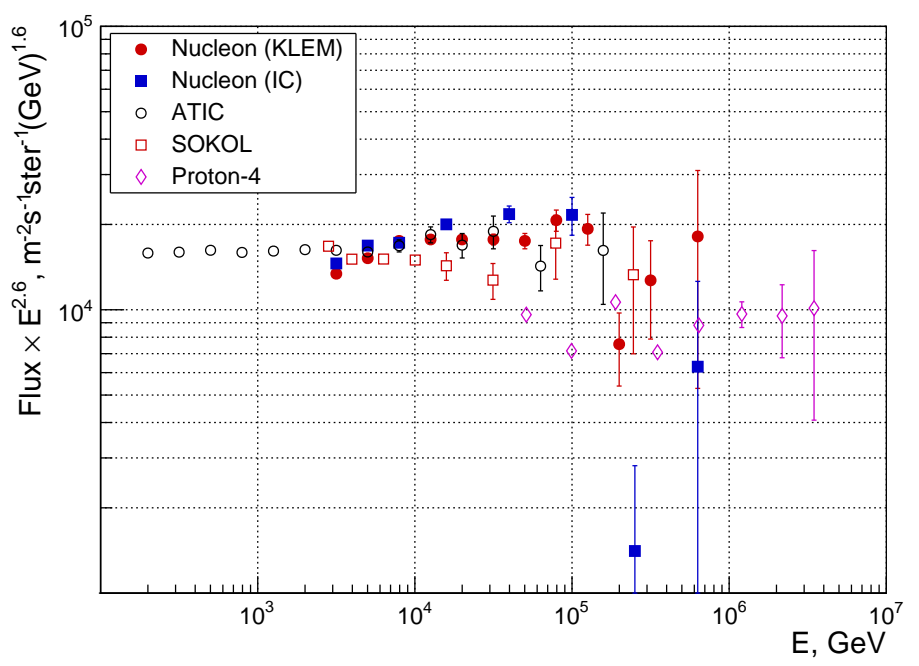

Figure 12: All-particle spectrum measured by NUCLEON with the KLEM and the calorimeter methods and data of PROTON-4 [4], SOKOL [8], ATIC [11].

\subsection{Secondary nuclei}

It is supposed that some nuclei of cosmic rays, like Be or B, have presumably secondary nature. 
They are products of spallation of the primary nuclei of cosmic rays, such as carbon, oxygen or iron with interstellar gas consisting in its main part of hydrogen and helium. Secondary nuclei are useful instrument to study propagation of cosmic rays in the Galaxy. The abundance of a secondary nucleus at some given energy expected to be approximately proportional to the lifetime of the parent primary nuclei in the Galaxy which itself depend on the nuclear cross section of the primary nuclei against the spallation process and on the diffusion coefficient describing the propagation of cosmic rays in the Galaxy at the same energy [46, Chapter 9]. The most useful quantities in this respect are various ratios of fluxes of secondary nuclei to primary nuclei.
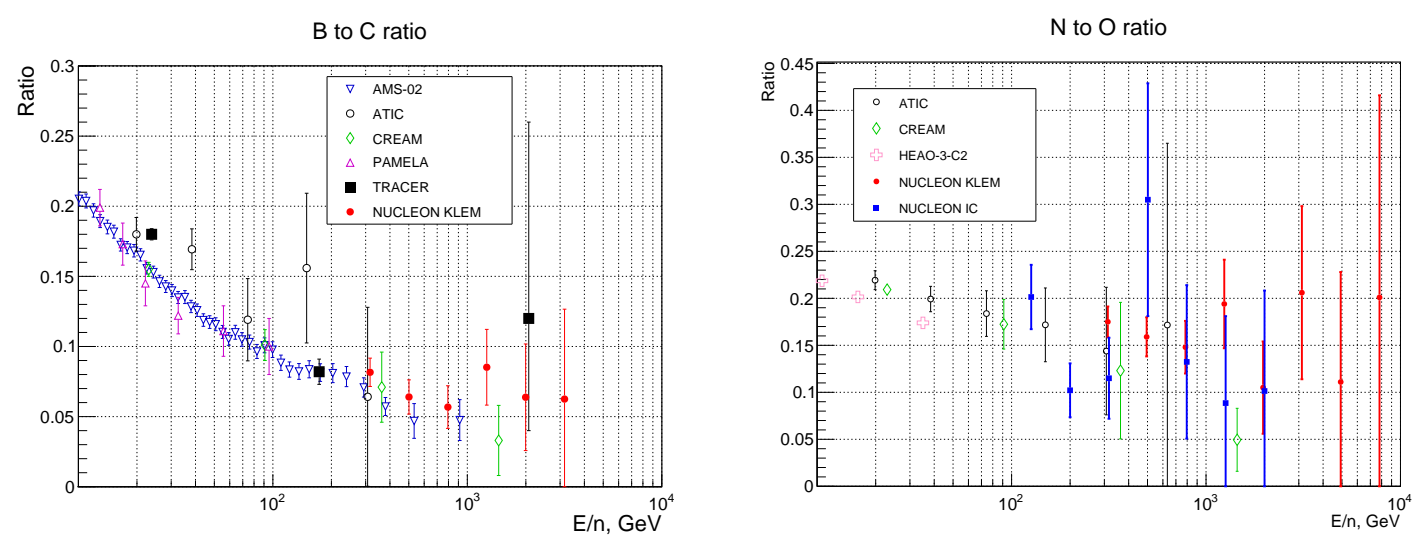

Figure 13: $B / C$ and N/O ratios measured by the NUCLEON spectrometer and in the experiments AMS-02 [47], ATIC [48], CREAM-I [49], PAMELA [50], HEAO-3-C2 [51], TRACER [41, 52].

Figure 13 shows the ratios $\mathrm{B} / \mathrm{C}$ and N/O measured in the present work and in other experiments: AMS-02 [47], ATIC [48], CREAM-I [49], PAMELA [50], HEAO-3-C2 [51], TRACER $[41,52]$. For B/C ratio only KLEM data are shown since the statistics of the calorimeter are too low yet. Boron to carbon and nitrogen to oxygen ratios of the NUCLEON spectrometer are in reasonable agreement with the data of other experiments in the energy regions where they can be compared, but the NUCLEON experiment provides the data points at hight energies, unavailable in previous experiments.

It is expected that boron to carbon ratio is a decreasing function of energy. It was confirmed generally in all previous experiments except one point at the highest energy ( $2 \mathrm{TeV} /$ nucleon) of the TRACER experiment $[41,52]$ which gives a sign of increasing of the ratio, but the statistical error is high. The last three points of the NUCLEON spectrometer also give an indication of increasing the ratio, but the statistical reliability is not high yet. The data taking of NUCLEON is continued and the statistics increases.

Similar indications is seen in the N/O ratio (figure 13, right panel). It is expected for a part of nitrogen nuclei flux to be primary nature but an essential fraction of the flux expected to be secondary nuclei. Therefore it is expected for N/O ratio to be decreasind function of energy. The statistics of N/O ratio is not high in the NUCLEON data but there is no clear indication of decreasing of the ratio with energy.

Note that there are models predicting an increasing in the ratios of secondary nuclei to the primary ones at sufficiently high energies [53]. It is assumed in these models that a significant part of the secondary nuclei is formed already at the stage of cosmic ray acceleration, inside the 
termination shock of the supernova remnant. Such secondary nuclei can be accelerated in the process of expansion of the supernova shell, and this acceleration can produce an excess in the ratio for such nuclei at high energies. Perhaps the signs of the existence of such a mechanism for the acceleration of secondary nuclei are already observed in the data of the NUCLEON experiment. An increasing of the statistics should clarify this issue.

Among the cosmic ray nuclei between silicon and iron there is a rather large fraction of secondary nuclei produced by spallation of the primary iron nuclei in inelastic collisions with interstellar gas. Therefore one might expect that a ratio of flux of some nucleus from this region of nuclei to the flux of the iron was a decreasing function. $\mathrm{Ar} / \mathrm{Fe}$ and $\mathrm{Ca} / \mathrm{Fe}$ ratios were measured in the HEAO-3-C3 heavy nuclei experiment up to energies about $600-700 \mathrm{GeV} /$ nucleon in 19985-1987 $[54,55,56]$. Unexpected increasing of the ratios was observed above the energy $100 \mathrm{GeV} /$ nucleon, but the authors questioned the reality of the phenomenon and tied it to a possible systematic error in the energy measurement procedure. Later the ratio of the flux of all nuclei with charges from 16 to 24 to the flux of iron was measured in the ATIC experiment [42] and the effect was qualitatively confirmed for this combined ratio.

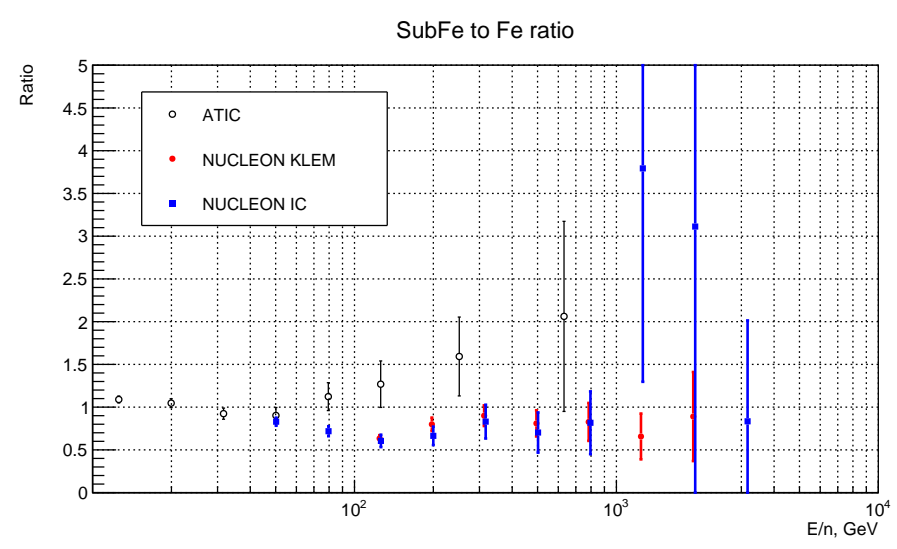

Figure 14: Ratios of the spectra of nuclei with charges $Z=16 \div 24$ in terms of energy per nucleon to the spectrum of iron nuclei for the NUCLEON experiment and the ATIC experiment [42].

Ratios of the spectra of nuclei with charges $Z=16 \div 24$ in terms of energy per nucleon to the spectrum of iron nuclei for the NUCLEON experiment and the ATIC experiment [42] are shown in figure 14. The calorimeter method data qualitatively confirm the results from the ATIC experiment, but the statistical errors of the calorimeter method are large, as well as the statistical errors of the ATIC experiment. The KLEM method, although not explicitly showing the theoretically expected decrease of the $Z=16 \div 24 / \mathrm{Fe}$ ratio with energy, which is already important, does not show the growth of this ratio similar to the results and outcomes of the ATIC experiment and the calorimeter method of the NUCLEON experiment. It is difficult to talk about systematic differences between the results of the calorimeter and the KLEM methods of the NUCLEON experiment, because all the differences occur within the statistical uncertainty. The situation expected to become clearer with a larger set of data in the NUCLEON experiment. 


\section{Conclusion}

A preliminary analysis of two years data of the NUCLEON space experiment gives multiple indications of the existence of a number of interesting features in the energy spectra of cosmic ray nuclei at energies from a few $\mathrm{TeV}$ to $\sim 100 \mathrm{TeV}$ (per particle). A number of questions are posed by the data which may be clarified with growing up of the statistics of the NUCLEON experiment. The NUCLEON space experiment is continuing and is in fact in its initial phase since no more than $1 / 3$ expected statistics have been collected yet. Increasing of the statistics and improving the quality of the data is expected.

\section{Acknowledgements}

We are grateful to ROSCOSMOS State Space Corporation and Russian Academy of Sciences for their continued support of this research.

\section{References}

[1] N. L. Grigorov, V. S. Murzin and I. D. Rapoport, Journal of Experimental and Theoretical Physics, 34 (1958) 506-507 )in Russian).

[2] V. S. Murzin, Progress on Elementary Partides and Cosmic Ray Physics, 1967.9 (1967) 245-302.

[3] V. V. Akimov, N. L. Grigorov, V. E. Nesterov, I. D. Rapoport, I. A. Savenko, G.A. Skuridin, A. F. Titenkov. 11th ICRC, 1969. Supplement to Volume 29. P.517-520.

[4] N. L. Grigorov, V. E. Nesterov, I. A. Savenko. Space Research XII, 2 (1972), 1617-1622.

[5] I. P. Ivanenko, I. D. Rapoport, V. Y. Shestoperov, et al., JETP Lett., 48 (1988) 510-513.

[6] I. P. Ivanenko, I. D. Rapoport, V. Ya. Shestoperov, et al. JETP Lett., 1989, 49 (1989) 222-224.

[7] P. I. Ivanenko, D. I. Rapoport, V. Ya. Shestoperov, et al., 21st ICRC, 1990, 3 77-80.

[8] I. P. Ivanenko, V. Ya. Shestoperov, L. O. Chikova et al., 23rd ICRC, 1993, 2 17-20.

[9] I. P. Ivanenko, V. Y. Shestoperov, D. M. Podorozhnyj, et al., Rossijskaya Akademiya Nauk, Izvestiya, Seriya Fizicheskaya, 57 (1993) 76-79.

[10] A. D. Panov, J. H. Adams, H. S. Ahn et al., Bulletin of the Russian Academy of Sciences: Physics 71 issue 4 (2007) 494-497, [arXiv : astro-ph / 0612377 ]

[11] A. D. Panov, J. H. Adams, H. S. Ahn et al., Bulletin of the Russian Academy of Sciences: Physics 73 (2009) 564-567, [arXiv:1101.3246].

[12] A.D. Panov, N.V. Sokolskaya and V.I. Zatsepin. ApJ, 837 (2017) 77.

[13] J. Chang, J. H. Adams Jr, H. S. Ahn, et al. Nature 456 (2008) 362-365.

[14] A.D. Panov, V.I. Zatsepin, N.V. Sokolskaya, et al.Astrophys. Space Sci. Trans., 7 (2011) P. 119-124.

[15] Y.S. Yoon, T. Anderson, A. Barrau et al., ApJ 839 (2017) 8, [arXiv: 1704.02512 [astr-ph.HE] ].

[16] H. S. Ahn, P. Allison, M. G. Bagliesi et al., ApJ Letters 714 (2010) L89-L93, [arXiv: 1004.1123 [astro-ph.HE] ]

[17] M. Aguilar, D. Aisa, B. Alpat, A. Alvino et al. (AMS-02 Collaboration), PRL 114 (2015) 171103. 
[18] S. Haino, K. Abe, K. Anraku, et al. 28th ICRC, 2003, P.1825-1828..

[19] S. Haino, T. Sanuki, K. Abe, et al. Phys. Lett. B. 594 (2004),35-46.

[20] T. Sanuki, for the BESS Collaboration, Nucl. Phys. B Suppl., 145 (2005) 132-135.

[21] M. Boezio, V. Bonvicini, P. Schiavon, et al. Astropart.Phys. 19 (2003) 583-604.

[22] . O. Adriani, G.C. Barbarino, G.A. Bazilevskaya, et al., Science, 332 Issue 6025 (2011) 69-72.

[23] Y.S. Yoon, H.S. Ahn, T. Anderson, et al. 31st ICRC, 2009, ID0668.

[24] Y.S. Yoon, H.S. Ahn, P.S. Allison, et al. ApJ, 728 (2011) 122.

[25] A. Ya. Varkovitskaya, E. A. Zamchalova, V. I. Zatsepin, T.V. Lazareva, G. P. Sazhina, N.V. Sokol'skaya, JETP Lett, 57 (1993) 469-472

[26] V I. Zatsepin, T. V. Lazareva, G. P. Sazhina, N. V Sokol'skaya, Physics of Atomic Nuclei, 57, Issue 4 (1994) 645-650.

[27] K. Asakimori, T.H. Burnett, M.L. Cherry, et al., ApJ, 502 (1998) 278-283.

[28] M. Hareyama, V.A. Derbina, V.I. Galkin, et al., ApJ, 628 (2005) L41-L44.

[29] J. Adams, G. Bashindzhagyan, A. Chilingaryan, et al., in AIP Conference Proceedings, vol. 504, pp. 175-180, 2000. DOI.

[30] J. Adams, G. Bashindzhagyan, P. Bashindzhagyan et al., Advances in Space Research 27 (2001) 829-831.

[31] N. A. Korotkova, D. M. Podorozhnyi, E. B. Postnikov et al., Physics of Atomic Nuclei 65 (2002) $852-860$.

[32] G. L. Bashindzhagyan, A. G. Voronin, S. A. Golubkov et al., Instruments and Experimental Techniques 48 (2005) 32-36.

[33] D. M. Podorozhnyi, E. B. Postnikov, L. G. Sveshnikova and A. N. Turundaevsky, Physics of Atomic Nuclei 68 (2005) 50-59.

[34] G. Voronin, V. M. Grebenyuk, D. E. Karmanov et al., Instruments and Experimental Techniques 50 (2007) 187-195.

[35] G. Voronin, V. M. Grebenyuk, D. E. Karmanov et al., Instruments and Experimental Techniques 50 (2007) 176-187.

[36] M. Podorozhnyi, V. L. Bulatov, N. V. Baranova et al., Bulletin of the Russian Academy of Sciences 71 (2007) 500-502.

[37] V. L. Bulatov, A. V. Vlasov, N. V. Gorbunov et al., Instruments and Experimental Techniques 53 (2010) 29-35.

[38] E. Atkin, V. Bulatov, V. Dorokhov et al., Nuc. Instr. Meth. A 770 (2015) 189-196.

[39] E. Atkin, V. Bulatov, V. Dorokhov et al., Journal of Cosmology and Astroparticle Physics, 2017 issue 07 (2017) 020, [arXiv: 1702.02352 [astro-ph.HE]].

[40] V.I. Zatsepin, J.H. Adams, Jr., H.S. Ahn, et al., Bulletin of the Russian Academy of Sciences. Physics, 68(11) (2004) 1780-1783.

[41] A. Obermeier, M. Ave, P. Boyle, C. Höppner, J. Hörandel and D. Müller, ApJ 742 (2011) 14, [arXiv:1108.4838]. 
[42] A. D. Panov, N. V. Sokolskaya, and V. I. Zatsepin, Nuclear Physics B (Proc. Suppl.) 256-257 (2014) 233-240.

[43] M. Ave, P. J. Boyle, F. Gahbauer et al., ApJ 678 (2008) 262-273.

[44] A. D. Panov, V. I. Zatsepin, and N. V. Sokolskaya, Bulletin of the Russian Academy of Sciences. Physics 77 No 5 (2013) 613-615,

[45] V. I. Zatsepin and N. V. Sokolskaya, A\&Astrophysics 458 (2006) 1-5, [arXiv: 1108.4838 ].

[46] T. K. Gaisser. Cosmic rays and particle physics. Cambridge University Press, Cambridge, New York, Port Chester, Sydney: 1990.

[47] . M. Aguilar, D. Aisa, B. Alpat, et al. (AMS-02 Collaboration). PRL 117 (2016) 231102.

[48] A. D. Panov, N. V. Sokolskaya, J. H. Adams, et al. 30th ICRC, 2008, 2 3-6.

[49] H.S. Ahn, P.S. Allison, M.G. Bagliesi, et al. Astropart. Phys. 30 (2008) 133-141.

[50] O. Adriani, G. C. Barbarino, G. A. Bazilevskaya, et al. 2014 ApJ 791 (2014) 93.

[51] J.J. Engelmann, P. Ferrando, A. Soutoul, et al. A\&A, 233 (1990) 96-111.

[52] . A. Obermeier, P. Boyle, J. Hörandel, D. Müller, ApJ, 752 (2012), 69.

[53] E. G. Berezhko, L. T. Ksenofontov, V. S. Ptuskin, V. N. Zirakashvili, H. J. Voelk. A\&A 410 (2003) 189-198.

[54] M.D. Jones, J. Klarmann, E.C. Stone, C.J. Waddington, W.R. Binns, T.L. Garrard, M.H. Israel. 19th ICRC, 1985, 2 28-31.

[55] M.H. Israel, M.D. Jones, M.P. Kamionkowski, J. Klarmann, E.C. Stone, C.J. Waddington, W.R. Binns, T.L. Garrard, 20th ICRC, 1987, 1 330-333

[56] W.R. Binns, T.L. Garrard, M.H. Israel, M.D. Jones, M.P. Kamionkowski, J. Klarmann, E.C. Stone, C.J. Waddington, ApJ, 324 (1988) 1106-1117. 